\title{
Frequency and phenotypic characteristics of RPE65 mutations in the Chinese population
}

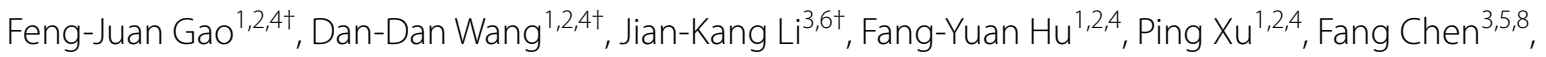 \\ Yu-He Qi ${ }^{1}$, Wei Liu' ${ }^{1}$, Wei Li, ${ }^{3,7}$, Sheng-Hai Zhang ${ }^{1,2,4}$, Qing Chang ${ }^{1,2,4}$, Ge-Zhi Xu $u^{1,2,4^{*}}$ and Ji-Hong Wu $u^{1,2,4^{*}}$ (D)
}

\begin{abstract}
Background: The retinoid isomerohydrolase RPE65 has received considerable attention worldwide since a successful clinical gene therapy was approved in 2017 as the first treatment for vision loss associated with RPE65-mediated inherited retinal disease. Identifying patients with RPE65 mutations is a prerequisite to assessing the patients' eligibility to receive RPE65-targeted gene therapies, and it is necessary to identify individuals who are most likely to benefit from gene therapies. This study aimed to investigate the RPE65 mutations frequency in the Chinese population and to determine the genetic and clinical characteristics of these patients.
\end{abstract}

Results: Only 20 patients with RPE65 mutations were identified, and RPE65 mutations were determined to be the 14th most common among all patients with genetic diagnoses. Ten novel variants and two hotspots associated with FAP were identified. A literature review revealed that a total of 57 patients of Chinese origin were identified with pathogenic mutations in the RPE65 gene. The mean best Snellen corrected visual acuity was worse (mean $1.3 \pm 1.3$ LogMAR) in patients older than 20 years old than in those younger than 15 years old ( $0.68 \pm 0.92$ LogMAR). Bone spicule-like pigment deposits (BSLPS) were observed in six patients; they were older than those without BSLP and those with white-yellow dots. Genotype-phenotype analysis revealed that truncating variants seem to lead to a more severe clinical presentation, while best corrected visual acuity testing and fundus changes did not correlate with specific RPE65 variants or mutation types.

Conclusions: This study provides a detailed clinical-genetic assessment of patients with RPE65 mutations of Chinese origin. These results may help to elucidate RPE65 mutations in the Chinese population and may facilitate genetic counseling and the implementation of gene therapy in China.

Keywords: Inherited retinal dystrophy, RPE65 gene mutations, Next-generation sequencing, Chinese population, Genotype-phenotype correlations

\section{Background}

The retinoid isomerohydrolase RPE65 has received considerable attention worldwide since a successful clinical gene therapy was approved in 2017 as the first treatment for vision loss associated with RPE65-mediated inherited

\footnotetext{
*Correspondence: proxgzeent@163.com; jihongwu@fudan.edu.cn ${ }^{\dagger}$ Feng-Juan Gao, Dan-Dan Wang and Jian-Kang Li have contributed equally to this work.

${ }^{1}$ Eye Institute, Eye and ENT Hospital, College of Medicine, Fudan University, Shanghai 200032, China

Full list of author information is available at the end of the article
}

retinal disease (IRD) (https://www.fda.gov/NewsEvents/ Newsroom/Press Announcements/ucm589467.htm). Identifying patients with RPE65 mutations is a prerequisite to assessing the patients' eligibility in receiving RPE65-targeted gene therapies, and it is necessary to identify individuals who are most likely to benefit from gene therapies.

To date, nearly 200 disease-causing mutations in the RPE65 gene have been reported (Human Gene Mutation Database (HGMD); professional version 2019.2), which are associated with a large heterogeneous group 
of retinal dystrophies, including Leber congenital amaurosis (LCA) type 2, early onset severe retinal dystrophy, retinitis pigmentosa (RP) type 20 and fundus albipunctatus (FAP). Studies have shown that the mutation frequency and phenotypic variation of RPE65 varies notably between different ethnic groups. For example, RPE65 mutations are thought to affect approximately 1000 to 3000 people in the United States (Population clock. 2018. Available: https://www.census.gov/popclock/ [Accessed 14 Aug 2018]), while 6\% of all LCA cases in Caucasians [1], $16 \%$ in the Danish LCA cohort [2], and only a few LCA cases were reported in Chinese populations $[3,4]$. Generally, most studies associated with RPE65 mutations were performed in Western populations $[2,5,6]$, and the exact frequency of RPE65 mutations in all forms of IRD and the variety of associated phenotypes in China has not been determined.

In the current study, we performed a comprehensive mutation analysis in 1434 IRD patients. Twenty patients with RPE65 mutations were identified, and their specific clinical phenotypes were presented. Moreover, we further reviewed the varied phenotypes and genotypes of all cases of RPE65 mutations of Chinese origin reported in the literature. These results provide a brief overview of the frequency and phenotypic characteristics of the RPE65 mutation in the Chinese population.

\section{Methods}

\section{Subjects, ethics statement and NGS analysis}

A total of 1434 Chinese patients with IRDs and their available family members (total participants: 3576) were recruited from the eye genetic disease clinic of the Eye and ENT Hospital of Fudan University between January 2017 and June 2019. Of these, 956 patients had been mentioned in our previous report [Gao and others 2019]. Written informed consent in accordance with the tenets of the Declaration of Helsinki was obtained from all participants or their guardians. This study was approved by the Ethics Committee of the Eye and ENT Hospital of Fudan University. DNA was isolated from peripheral blood using the FlexiGene DNA Kit (Qiagen, Venlo, the Netherlands) according to the manufacturer's protocol. NGS analysis and bioinformatics analysis were performed as previously reported [7]. We designed a high-throughput targeted enrichment approach to exoncapture regions of 586 genes that are involved in common inherited eye diseases. The probe length of the panel is 90 $\mathrm{nt}$, the total target area obtained is $2.3 \mathrm{M}$. On average, the mean coverage depth was more than 400X, and the coverage of target region was $99.9 \%$ using BGISEQ-2000. Then the sequence data obtained were analyzed as previously reported [7]. Previous reported variants were determined using Human Gene Mutation Database (HGMD, professional version 2019.2). For variants that passed the initial filtration, Sanger sequencing was carried out for segregation analysis and variants validation.

\section{Clinical evaluations}

All patients with pathogenic mutations in RPE65 underwent a full ophthalmic examination, including best Snellen corrected visual acuity testing (BCVA, they were converted to equivalent value of logarithm of minimal angle of resolution (logMAR) unit), slit lamp biomicroscopy, tonometry, fundus examination, wide-field fundus imaging (Optos PLC, Dunfermline, United Kingdom), swept-domain optical coherence tomography (SD-OCT, Spectralis HRA+OCT, Heidelberg, Engineering Inc., Heidelberg, Germany), visual field (Humphrey Visual Field Analyzer, Carl Zeiss Inc., CA, USA), and full-field electroretinography (ERG, according to the standards of the International Society for Clinical Electrophysiology of Vision; available at www.iscev.org).

\section{Results \\ Genetic analyses}

Of the 1434 patients with IRD, $74.55 \%$ of patients $(n=1069)$ received a genetic diagnosis, and a total of 1516 variants involved 87 genes were identified. Only 41 alleles representing 26 distinct variants in 17 families (20 patients: 10 males, 10 females) were identified in the RPE65 gene (NM_000329.2, Table 1 and Additional file 5: Table S1), accounting for $2.83 \%$ of all the variants, and the gene was ranked as the seventh most common gene detected in this cohort of patients with IRD (Additional file 1: Figure S1A and S1B). However, the number of patients with RPE65 mutations only accounted for $1.87 \%(20 / 1069)$ of all patients with genetic diagnoses and was the 14th most common among all the patients (Additional file 1: Fig. 1c, d). Pedigrees and mutations of the 17 families are available in Additional file 2: Figure S2. Of the 26 distinct variants identified in this study, 10 (c.1039C $>$ T p.Arg347Cys, c.1255C $>$ T p.Pro419Ser, c.1444G>A p.Asp482Asn, c.334T>A p.Cys112Ser, c.3542A>G, c.376del p.Val126*fs1， c.806_809delinsTGG AGCCATGAAG p.SerLeu269MetGluProTer, c.837del p.Phe279Leufs46, c.886del p.Arg296*fs1, c.94+2T>A) were novel (Additional file 3: Figure S3), including seven likely pathogenic variants and three missense variants of uncertain significance (p.Pro419Ser, p.Cys112Ser and p.Arg347Cys), which are localized in highly conserved residues (Additional file 4: Figure S4A). Bioinformatics analysis results of the novel variants are shown in Additional file 6: Table S2. Of the 16 variants reported previously, p.His68Tyr and c.998+1G>A were firstly reported to be associated with LCA. 
Table 1 RPE65 variants identified in this cohort of patients

\begin{tabular}{|c|c|c|c|c|c|c|}
\hline Nucleotide change & Amino acid change & Mutation type & Exon/intron & Patients & ACMG category & References \\
\hline c. $1399 C>G$ & p.Pro467Ala & Missense & E13 & F1-1, F3-1 & $P$ & {$[8,13]$} \\
\hline$c .272 \mathrm{G}>\mathrm{A}$ & p.Arg91Gln & Missense & E4 & $F 2-1$ & $P$ & [14-16] \\
\hline$c .271 C>T$ & p.Arg91Trp & Missense & E4 & F2-1, F16-1 & $P$ & {$[17,18]$} \\
\hline c.1338G > T & p.Arg446Ser & Missense & E12 & F5-1, F7-1, F7-2 & $P$ & {$[4,19]$} \\
\hline c. $1543 C>T$ & p.Arg515Trp & Missense & E14 & F6-1, F6-2 & $P$ & {$[20,21]$} \\
\hline c. $1444 G>A$ & p.Asp482Asn & Missense & E13 & F6-1, F6-2, F17-1 & $L P$ & Novel \\
\hline c. $1255 C>T$ & p.Pro419Ser & Missense & E12 & F8-1 & VUS & Novel \\
\hline$c .202 C>T$ & p.His68Tyr & Missense & E3 & F8-1 & P & {$[22,23]$} \\
\hline c. $1590 C>A$ & p.Phe530Leu & Missense & E14 & F9-1 & $P$ & {$[4,24]$} \\
\hline c.997G $>C$ & p.Gly333Arg & Missense & E9 & F10-1 & $P$ & {$[25]$} \\
\hline c.334T>A & p.Cys112Ser & Missense & E4 & F10-1 & VUS & Novel \\
\hline c. $131 \mathrm{G}>\mathrm{A}$ & p.Arg44GIn & Missense & E3 & F12-1 & P & {$[14,26]$} \\
\hline c. $200 T>G$ & p.Leu67Arg & Missense & E3 & F13-1 & $\mathrm{P}$ & {$[27,28]$} \\
\hline c. $1304 A>G$ & p.Tyr435Cys & Missense & E12 & F13-1 & $P$ & {$[29]$} \\
\hline c. $1039 C>T$ & p.Arg347Cys & Missense & E10 & F14-1 & VUS & Novel \\
\hline c.1078G >C & p.Ala360Pro & Missense & E10 & F14-1 & LP & {$[30]$} \\
\hline$c .493 C>T$ & p.Gln $165^{*}$ & Nonsense & E5 & F4-1, F4-2 & $P$ & [4] \\
\hline c.1380G >A & p.Trp460* & Nonsense & E13 & F15-1 & $P$ & {$[31]$} \\
\hline$c .94+2 \mathrm{~T}>\mathrm{A}$ & - & Splicing & 12 & F1-1 & LP & Novel \\
\hline$c .998+1 \mathrm{G}>\mathrm{A}$ & - & Splicing & 110 & F3-1 & $P$ & [13] \\
\hline$c .354-2 A>G$ & - & Splicing & 15 & F7-1, F7-2 & $L P$ & Novel \\
\hline c.858+1del & - & Splicing & 19 & F9-1, F12-1 & $P$ & {$[8]$} \\
\hline c.376del & p.Val126fs*1 & Frameshift & EX5 & F16-1 & $L P$ & Novel \\
\hline $\begin{array}{l}\text { c.806_809delinsTGG } \\
\text { AGCCATGAAG }\end{array}$ & p.SerLeu269MetGluProTer & Frameshift & EX8 & F17-1 & $P$ & Novel \\
\hline c.837del & p.Phe279Leufs*46 & Frameshift & EX8 & F11-1 & $L P$ & Novel \\
\hline c.886del & p.Arg296fs & Frameshift & EX9 & F15-1 & LP & Novel \\
\hline
\end{tabular}

F: family; E: Exon; I: Intron; P: Pathogenic; LP: Likely pathogenic; VUS: variants of uncertain significance

To date, a total of 39 patients from 27 unrelated Chinese families have been reported with pathogenic mutations in RPE65 (Additional file 5: Table S1). Together with the 20 patients (two have been previously reported: F4-1 and F5-1) in this study [8], 57 patients of Chinese origin were diagnosed. Of the 115 variants identified in these patients, the majority of pathogenic defects $(71.3 \%$, $\mathrm{n}=82)$ were missense variants, and $28.7 \%(\mathrm{n}=33)$ were nonsense, frameshift, or splice-site mutations that severely affected protein function (Fig. 1a). Variants were distributed from exon 2 to exon 14 (Fig. 1b).

\section{Phenotypic characterization}

Of the 20 patients in the 17 families, four were diagnosed with RP, one with FAP, and 15 with LCA (Table 2). The mean age at visit was $16.4 \pm 12.59$ years (range 3-49 years; median, 10 years). All accepted patients (LCA and RP) experienced poor vision at an early age. The mean BCVA with LCA patients was $0.82 \pm 0.92$ (range 3.00-0.40) LogMAR, and 90\% (27/30) of eyes had a BCVA worse than 0.52 LogMAR. Of patients younger than 15 years, the mean BCVA was $0.68 \pm 0.92$ (range 1.30-0.40) LogMAR, while for patients older than 20 years, the mean BCVA was worse $(\mathrm{p}<0.001$, mean $1.30 \pm 1.30$ (range 3.00-1.00) LogMAR. Of the four patients with RP, the mean BCVA was $0.37 \pm 1.05$ (range 0.52-0.22) LogMAR, while the FAP patient maintained better vision (0/0.05 LogMAR). Bone spicule-like pigment deposits (BSLPs) were observed in six patients from five families $(30 \%, 6 / 20$, Fig. 2a), and no typical deposits were seen in $70 \%$ of patients $(n=14$, Fig. $2 b)$. Interestingly, we observed that subjects with BSLP were older than those without BSLP $(\mathrm{p}<0.05,26.83 \pm 14.86$ vs. $11.93 \pm 8.65$ years, respectively) (Fig. $2 \mathrm{~d}$ ). Eight patients (40\%, mean age $9.0 \pm 3.74$ years) showed white-yellow dots (WYD) scattered in the periphery of the retina $(n=5)$ or perimacular area $(n=3$, Fig. $2 c)$; they were younger than those $(n=12$, mean age $21.33 \pm 14.09$ years $)$ without WYD $(\mathrm{p}<0.01)$ and younger than patients with BSLP ( $p<0.05$, Fig. 2d). Cone and rod responses on ERG 


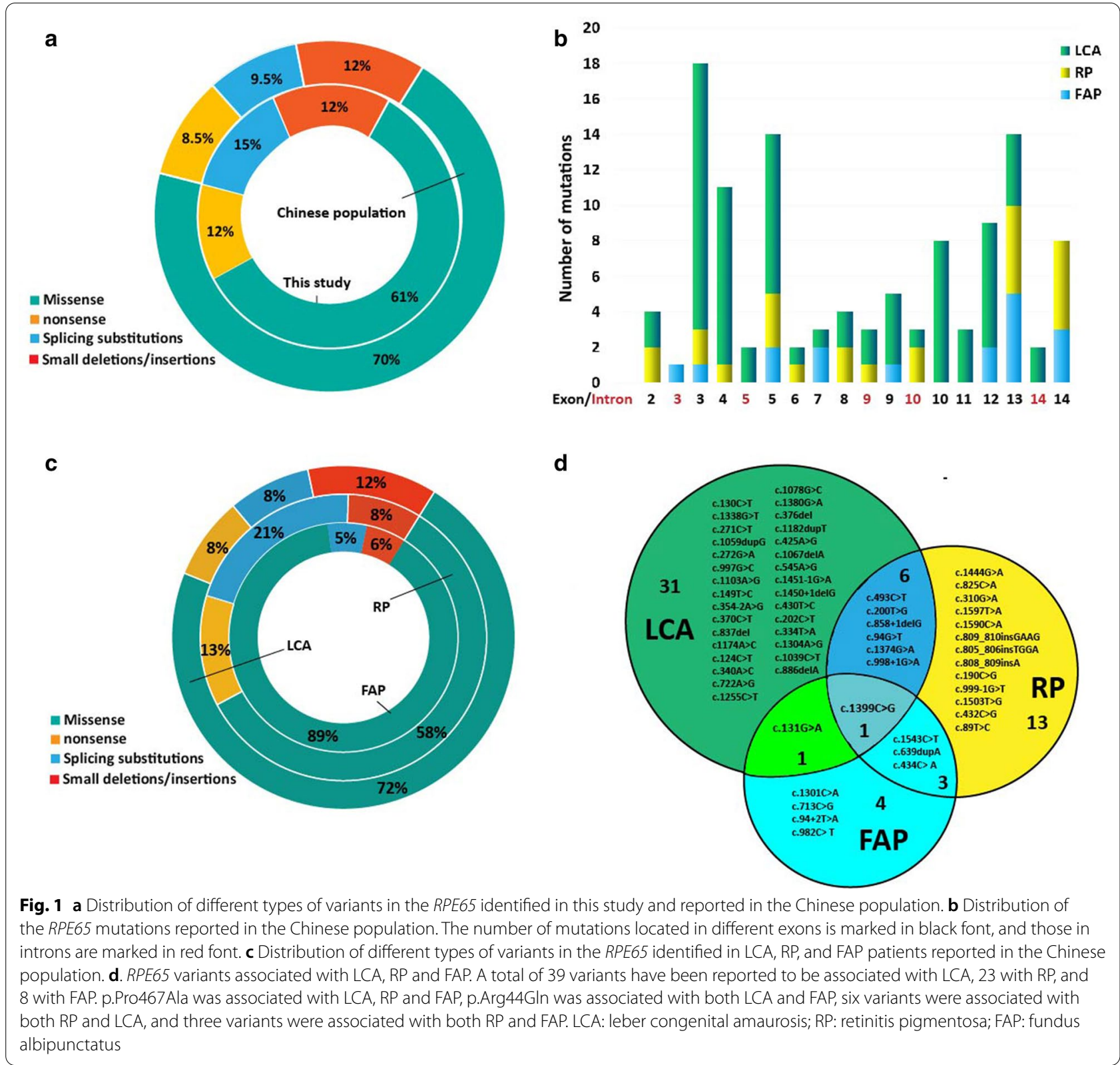

were nonrecordable in 14 of the 15 LCA patients and notably attenuated in the four RP patients.

\section{Genotype-phenotype correlations}

All RPE65 variants identified in the Chinese population are shown in Fig. 3 and Additional file 5: Table S1. To date, 39 variants have been reported to be associated with LCA, 21 with RP, and 8 with FAP. As no clinical features of the FAP patient with one frameshift were provided by Guoxing Yang et al. [9], the FAP diagnosis requires further confirmation, and this patient was not included in the statistical analysis. Of all the variants associated with
LCA $(\mathrm{n}=73), 71.2 \%(\mathrm{n}=52)$ were missense variants, and $28.8 \%(n=21)$ were nonsense $(n=5)$, frameshift $(n=9)$, or splice-site mutations $(\mathrm{n}=7)$ that severely affected protein function, while in the FAP group $(n=16), 93.75 \%$ $(\mathrm{n}=15)$ variants were missense, and only one splice-site mutation was identified (Fig. 1c). In the RP group, 58.3\% $(n=14)$ of variants were missense variants, $20.8 \%(n=5)$ were truncating stops, and $20.8 \%$ were frameshift $(n=2)$ or splicing defects $(n=3)$. Therefore, of all the variants $(\mathrm{n}=97)$ associated with LCA and RP, which have a severe early-onset clinical presentation, only $68 \%(n=66)$ were missense mutations, and the remaining $32 \%(n=31)$ of 
Table 2 Clinical characteristics of the 20 patients identified in this study

\begin{tabular}{|c|c|c|c|c|c|c|c|c|}
\hline Patients & Age (years) /sex & $\begin{array}{l}\text { BCVA LogMAR } \\
\text { R/L }\end{array}$ & $\begin{array}{l}\text { Refraction } \\
R / L\end{array}$ & $\begin{array}{l}\text { Age at disease } \\
\text { presentation } \\
\text { (years) }\end{array}$ & ERG & Fundus & Others & Diagnosis \\
\hline F1-1 & $12 / F$ & 0/0.05 & $+0.5 /+0.75$ & Congenital & $\begin{array}{l}\text { Undetectable rod ERG, } \\
\text { subnormal cone ERG }\end{array}$ & a & No & FAP \\
\hline$F 2-1$ & $6 / M$ & $0.60 / 0.82$ & $-0.5 /-2.0$ & Congenital & Extinct & $a, b$ & Nystagmus & LCA 2 \\
\hline F3-1 & $7 / \mathrm{M}$ & $0.40 / 0.40$ & $-4.0 /-3.75$ & Congenital & Extinct & $\mathrm{N}$ & Nystagmus & LCA 2 \\
\hline F4-1 & 15/M & $0.52 / 0.52$ & $-3.5 /-3.0$ & Congenital & Extinct & $a, b$ & Nystagmus & LCA 2 \\
\hline$F 4-2$ & $3 / F$ & $1.00 / 1.00$ & $+0.5 /+0.75$ & Congenital & Extinct & $\mathrm{N}$ & Nystagmus & LCA 2 \\
\hline F5-1 & $9 / F$ & $1.30 / 0.70$ & $+4.5 /+4.5$ & Congenital & Extinct & a & Nystagmus & LCA 2 \\
\hline F6-1 & 10/M & $0.22 / 0.40$ & $-/+0.5$ & 3 & $\begin{array}{l}\text { Profoundly attenuated } \\
\text { rod and cone ERGs }\end{array}$ & $\mathrm{N}$ & No & RP 20 \\
\hline F6-2 & 9/F & $0.40 / 0.40$ & $-/+0.25$ & 3 & $\begin{array}{l}\text { Profoundly attenuated } \\
\text { rod and cone ERGs }\end{array}$ & a & No & RP 20 \\
\hline F7-1 & 29/M & $1.00 / 3.00$ & - & Congenital & Extinct & b & Nystagmus & LCA 2 \\
\hline F7-2 & $31 / \mathrm{M}$ & $1.3 / 1.3$ & - & Congenital & Extinct & $b$ & Nystagmus & LCA 2 \\
\hline F8-1 & $7 / F$ & $1.00 / 0.13$ & $+1.5 /+2.25$ & Congenital & Extinct & a & Nystagmus & LCA 2 \\
\hline F9-1 & $11 / \mathrm{M}$ & $0.52 / 0.40$ & $-0.5 /-0.5$ & Congenital & $\begin{array}{l}\text { Profoundly attenuated } \\
\text { rod and cone ERGs }\end{array}$ & a & No & RP 20 \\
\hline F10-1 & $49 / F$ & $3.00 / 3.00$ & - & Congenital & Extinct & $b$ & Nystagmus & LCA 2 \\
\hline F11-1 & 20/M & $1.00 / 1.00$ & $-2.25 /-1.75$ & Congenital & $\begin{array}{l}\text { Profoundly attenuated } \\
\text { rod and cone ERGs }\end{array}$ & $\mathrm{N}$ & Nystagmus & LCA 2 \\
\hline F12-1 & $31 / F$ & $1.00 / 1.00$ & $-1.0 /-1.25$ & Congenital & Extinct & $b$ & Nystagmus & LCA 2 \\
\hline F13-1 & $9 / F$ & $0.52 / 0.40$ & $+2.5 /+2.25$ & Congenital & Extinct & $\mathrm{N}$ & $\begin{array}{l}\text { Nystagmus, oculo- } \\
\text { digital sign }\end{array}$ & LCA 2 \\
\hline F14-1 & $5 / F$ & $0.60 / 0.70$ & $+2.25 /+3.0$ & Congenital & Extinct & $\mathrm{N}$ & Nystagmus, esotropia & LCA 2 \\
\hline F15-1 & $5 / M$ & $0.82 / 1.00$ & $+2.0 /+2.25$ & Congenital & Extinct & $\mathrm{N}$ & Nystagmus & LCA 2 \\
\hline F16-1 & $30 / M$ & $2.00 / 2.00$ & $-1-$ & Congenital & Extinct & $\mathrm{N}$ & Nystagmus & LCA 2 \\
\hline F17-1 & $30 / F$ & $0.30 / 0.40$ & $-4.0 /-4.5$ & Congenital & $\begin{array}{l}\text { Profoundly attenuated } \\
\text { rod and cone ERGs }\end{array}$ & $\mathrm{N}$ & No & RP 20 \\
\hline
\end{tabular}

F: family; R: right; L: left; LP = 3 LogMAR; HM = 2 LogMAR; a. white or white-yellow dots; b. Bone-spicule-like pigment; deposits; $N$ : no a or b; LCA: Leber congenital amaurosis; RP: retinitis pigmentosa; FAP: fundus albipunctatus

variants were truncating mutations. Nevertheless, in the FAP group, which showed relatively mild symptoms, 93.8\% $(\mathrm{n}=15)$ of all the variants were missense. Of all the LCA and RP patients, $50 \%$ had missense + missense mutations, $35.4 \%$ had missense + nonsense/frameshift/ splice-site mutations, and $14.6 \%$ did not have any missense mutations. However, of the eight FAP patients, 7 were missense + missense, and only one was a missense + splice-site mutation.

Only one variant, c.1399C>G (p. Pro467Ala), was associated with LCA, RP and FAP (Fig. 1d). This mutation was located in a highly evolutionarily conserved region (Additional file 4: Figure S4A) and altered the corresponding amino acid from proline to alanine. The $3 \mathrm{D}$ structural model of these amino changes is portrayed in Additional file 4: Figure S4B. Of the eight FAP patients, five had the p. Pro467Ala mutation, and the other three had the p.Arg515Trp mutation. It is likely that the two variants were hotspots of FAP. c.131G>A
p.Arg44Gln was associated with both LCA and FAP; when the second mutated allele is a truncating mutation $(\mathrm{c} .858+1 \mathrm{del})$, the patients were likely to present an LCA diagnosis, while if the second mutated allele is a missense mutation (p.Arg515Trp), the patients were likely to present an FAP diagnosis. p.Arg515Trp, p.Ala145Asp and p.Ala214Serfs*20 were associated with both RP and FAP, while p.Ala434Glu, p.Ser238Cys, c. $94+2 \mathrm{~T}>\mathrm{A}$, and p. Leu328Phe were only associated with FAP (Fig. 1d). Further analysis of the relationship between BCVA and fundus changes with specific mutations revealed that there was no correlation between them. Different individuals, even those with the same mutation, were found to show different changes. For example, patient F6-2 showed white-yellow dots scattered in the periphery of the retina, while patient F6-1, who had the same mutations, did not display these dots. However, we can't rule out the possibility that these dots would disappear with age. 


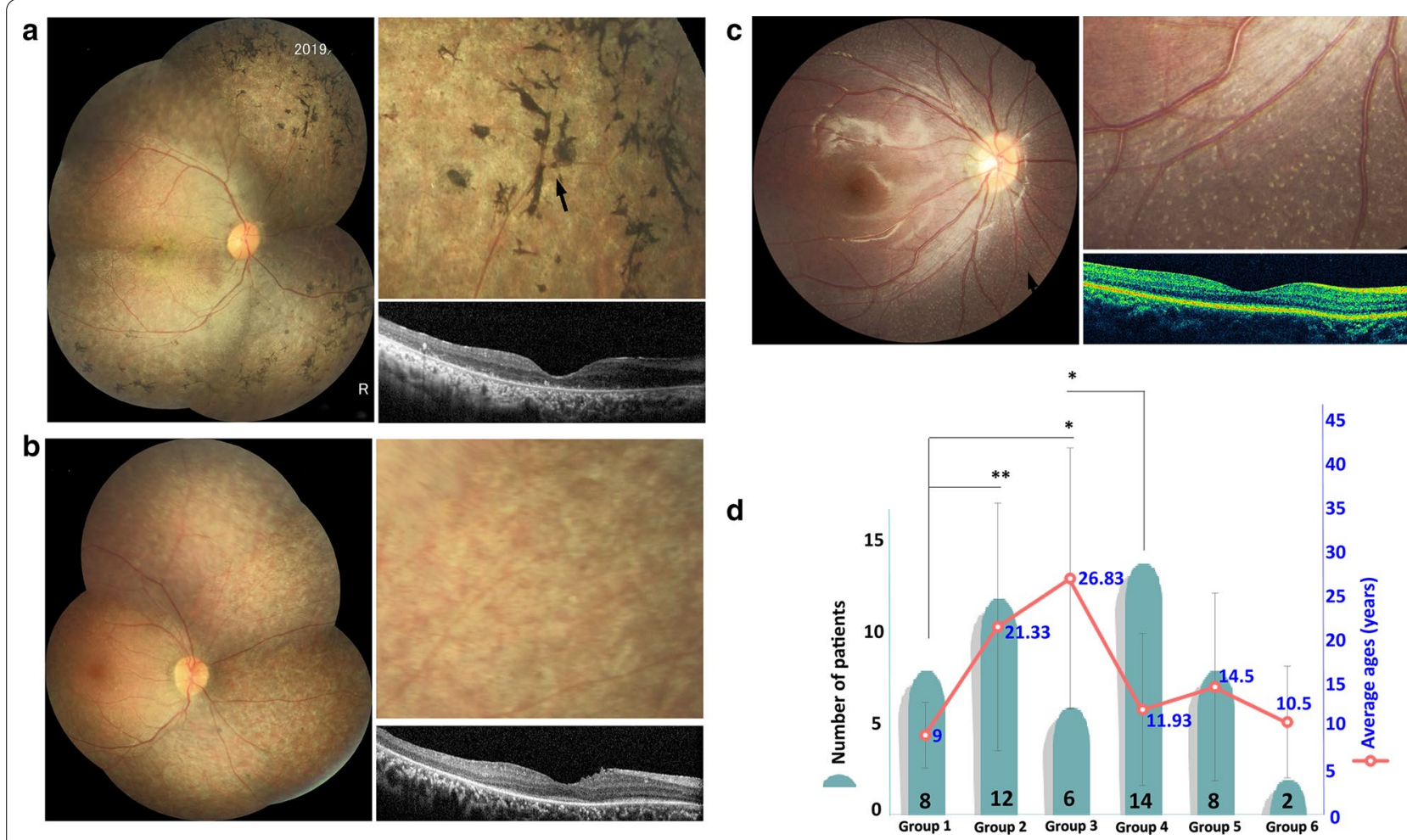

Fig. 2 a Color fundus photographs and spectral domain optical coherence tomography (SD-OCT) of patients showed bone spicule-like pigment deposits (BSLP). The image in the upper right is the corresponding enlarged figure showing BSLP in the fundus (black arrow). b Color fundus photograph and SD-OCT of patients without BDLP or white-yellow dots (WYD). The image in the upper right is the corresponding enlarged figure showing pigment dispersion in the mid-periphery. c Color fundus photograph and SD-OCT of patients with WYD (black arrow). The image in the upper right is the corresponding enlarged figure showing WYD scattered in the periphery of the retina. $\mathbf{d}$ Number of patients (black font) and their corresponding mean ages (blue font) in different groups. Group 1: patients with WYD. Group 2: patients without WYD. Group 3: patients with BDLP. Group 4: patients without BDLP. Group 5: patients without WYD and BDLP. Group 6: patients with WYD and BDLP. ${ }^{*} p<0.05$; **p $<0.01$

\section{Discussion}

RPE65 mutation-associated IRD usually occurs at an early age, and the majority of patients become fully blind in childhood or adulthood [10, 11]. Recent research has indicated that certain forms of RPE65-mediated IRDs are amenable to gene therapy. Achieving an in-depth understanding of RPE65 mutations and related phenotypic characteristics in the Chinese population is a prerequisite for developing RPE65-targeted gene therapies in China.

Only 20 patients with associated RPE65 mutations were identified from January 2017 to June 2019 in our hospital, accounting for $1.87 \%$ of all IRD patients and making RPE65 mutations the 14th most common among all patients. Together with other patients who have been reported, only 57 patients with RPE65 mutations have been identified in China [3, 8-16]. These results suggest that RPE65 mutations are rare in Chinese populations. As these mutations appear to be a low-probability, high-cost event, our data may provide strong clinical-based evidence for gene therapy researchers, economists, government policy makers, and ophthalmologists to make decisions in their corresponding work. The mean age of patients with RPE65 mutations was $16.4 \pm 12.59$ years (median, 10 years), and the mean BCVA was 0.82 \pm 0.92 LogMAR with LCA patients and $0.37 \pm 1.05$ LogMAR with RP patients. Of patients younger than 15 years, the mean BCVA was $0.68 \pm 0.92 \operatorname{LogMAR}$, while for patients older than 20 years, the mean BCVA was worse (mean $1.3 \pm 1.3 \operatorname{LogMAR}$ ). These data indicate that the optimal intervention window for subretinal gene therapy is within the first 2 decades of life.

El Matri, L. et al. reported that white dot deposits occurred in earlier stages, and clumped pigment occurred in later stages, in patients with RPE65 mutations [12]. A similar result was obtained in the current study: patients with RPE65 mutations are more likely to exhibit WYD in the first decade but show BSLP after the second decade of life. However, some patients (40\%) could not have BSLP or WYD, and these changes had no correlation with specific mutations or types or locations of mutations. 


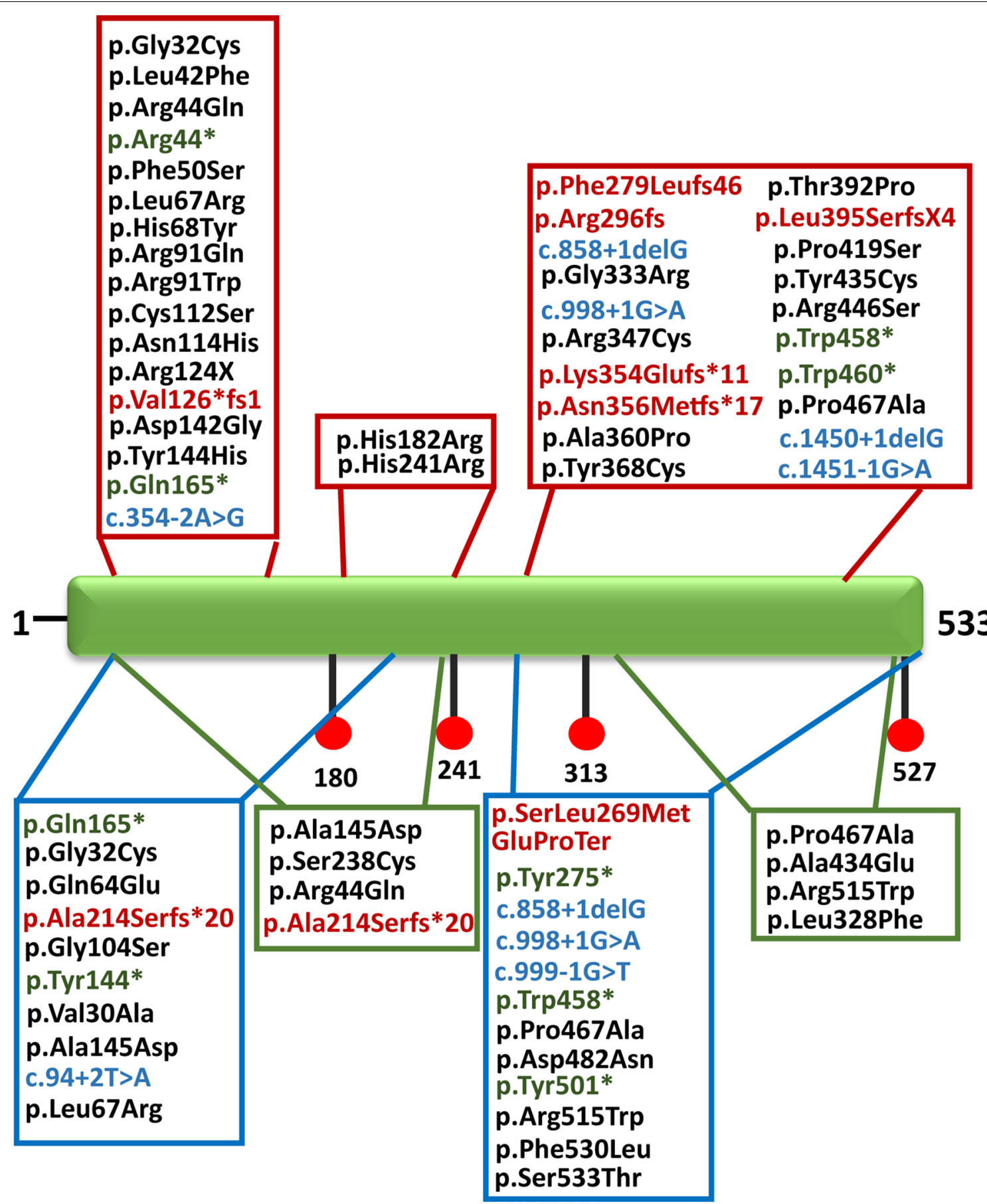

Fig. 3 Overview of RPE65 mutations reported to date in the Chinese population and this study. Blank: missense mutation. Blue: splicing mutation. Red: frameshift mutation. Green: nonsense mutation. Variants in the black box are mutations associated with LCA. Variants in the blue box are mutations associated with RP. Variants in the green box are mutations associated with FAP. LCA: leber congenital amaurosis; RP: retinitis pigmentosa; FAP: fundus albipunctatus

To date, genotype-phenotype correlations of patients with RPE65 mutations have not been highly distinct. It has not been determined why some mutations in RPE65 lead to LCA, while others lead to RP or FAP. In addition, the fact that most patients are compound heterozygotes hinders efforts to assess the effect of each mutation on the phenotypes and to evaluate possible allelic hierarchy. In this study, we found four notable cases of possible 
discordancy between clinical and molecular diagnosis. First, truncating variants seem to lead to a more severe clinical presentation (LCA or RP), while almost all FAP patients were caused by missense mutations. Second, two hotspots (p.Pro467Ala and p.Arg515Trp) associated with FAP were identified, helping to further elucidate the mutational spectrum of RPE65 in the Chinese population. Third, p.Pro467Ala was associated with LCA, RP and FAP, suggesting that this mutation may have a mild effect on protein function, and the phenotype is primarily affected by the second allele. Fourth, BCVA and fundus changes did not correlate with specific RPE65 variants or mutation types.

\section{Conclusions}

In the current study, we performed a comprehensive analysis of the phenotypes and genotypes of the 20 patients with RPE65 mutations identified in this study and all the RPE65 mutation cases of Chinese origin reported in the literature. Our data provide a brief overview of the frequency and phenotypic characteristics of RPE65 mutations in the Chinese population, help to characterize RPE65 mutations in China, and represent a possible reference for genetic counseling and the selection of eligible patients for gene augmentation.

\begin{abstract}
Abbreviations
ACMG: American College of Medical Genetics; BCVA: Best corrected visual acuity testing; BSLP: Bone-spicule-like pigment deposits; CFT: Central foveal thickness; CME: Cystoid macular edema; ERG: Full-field electroretinography; FAP: Fundus albipunctatus; HGMD: Human Gene Mutation Database; IRD: Inherited retinal disease; LCA: Leber congenital amaurosis; NGS: Next-generation sequencing; OMIM: Online Mendelian Inheritance in Man; RP: Retinitis pigmentosa; SD-OCT: Spectral domain optical coherence tomography; WYD: White-yellow dots.
\end{abstract}

\section{Supplementary Information}

The online version contains supplementary material available at https://doi. org/10.1186/s13023-021-01807-3.

Additional file 1: Figure S1. A. Distribution of inherited retinal disease (IRD)-causative genes variants $(n=1516)$ in 1069 patients. RPE65 mutations ( $n=41$ ) accounted for $2.70 \%$ of all the variants. B. Number of mutations of different IRD-causative genes. Only 41 variants were identified in the RPE65 gene, which ranked as the seventh most commonly detected gene in this cohort of patients with IRD. C. Percentage of IRD patients with variants in different causative genes; patients with RPE65 mutations only accounted for $1.87 \%$ of all patients $(n=1069)$. D. Number of IRD patients with different causative genes. Twenty patients with RPE65 mutations were identified, and they were placed as the 14th among all the patients.

Additional file 2: Figure S2. Pedigrees of the 17 families with RPE65 mutations. Filled symbols signify patients. Unfilled symbols represent unaffected family members. Arrows: probands. Square: male individuals; Circle: female individuals. A slash indicates a deceased person.

Additional file 3: Figure S3. Sequencing results of the ten novel RPE65 variants identified in this study. Arrows indicate the position of the mutated nucleotide. Mutation c.806_809delinsTGGAGCCATGAAG,
c.493C > T, c.354-2A>G, c.1255C > T, c.334T >A and c.1444G > A were shown in reverse strand.

Additional file 4: Figure S4. A. Multiple sequence alignment of different species of RPE65 mutations (c.1255C>T p.Pro419Ser, c.334T>A p.Cys112Ser, c.1039C > T p. Arg347Cys, and c.1399C > G p. Pro467Ala), the red arrow represents mutation sites. B. 3D structural model of the wild-type and mutant residues with RPE65 c.1399C>G p. Pro467Ala (black arrow).

Additional file 5: Table S1. Genotypes of the 57 patients from 50 families of Chinese origin reported previously.

Additional file 6: Table S2. Bioinformatic analysis results of the novel variants. Results of Fathmm can be found at: http://fathmm.biocompute. org.uk/fathmm-xf/cgi-bin/results.cgi?session=eb039a07-6c14-4e0d-97e3$5 \mathrm{~d} 2$ bfe729b4b, and http://fathmm.biocompute.org.uk/fathmm-xf/cgi-bin/ results.cgi?session $=5$ b257644-dd4b-4d56-b759-8c894fabd033.

\section{Acknowledgements}

The author would like to thank all the participants and the staffs for their valuable contribution to this research.

\section{Authors' contributions}

Ji-Hong Wu conceived and designed the experiments. Wei Liu, Qing Chang, Ping Xu, Ji-Hong Wu, Feng-Juan Gao, Dan-Dan Wang and Yu-He Qi collected the clinical samples. Feng-Juan Gao, Ji-Hong Wu, Fang-Yuan Hu, Jiang-Kang Li, Fang Chen, Wei Li and Dan-Dan Wang analyzed sequencing data. Ge-Zhi Xu, Wei Liu, Feng-Juan Gao and Yu-He Qi recruited patients, performed clinical examination of patients and clinical interpretation. Feng-Juan Gao and JiHong Wu drafted and revised the manuscript. All authors read and approved the manuscript. All authors read and approved the manuscript.

\section{Funding}

Supported by the National Natural Science Foundation of China (Grant NSFC81770925, 81790641, the Non-profit Central Research Institute Fund of Chinese Academy of Medical Sciences 2018PT32019). Program of Shanghai Academic Research Leader (20XD1401100). Program for Outstanding Medical Academic Leader (2019LJ01). Clinical Research Plan of SHDC (SHDC2020CR2041B). Xuhui district health and family planning commission key disease joint project (XHLHGG201807). Shanghai municipal science and technology major projects (2018SHZDZX05). Shanghai clinical medical center of ocular disease (2017ZZ01020).

\section{Availability of data and materials}

Please contact authors for data requests.

\section{Declarations}

Ethics approval and consent to participate

The study was in accordance with the Declaration of Helsinki and approved by the Ethics Committee of the Eye and ENT Hospital of Fudan University.

\section{Consent for publication}

Written informed consent was obtained from all the subjects or their guardians.

\section{Competing interests}

None of the authors has any conflicting interests to disclose. There are no financial disclosures in this article.

\section{Author details}

${ }^{1}$ Eye Institute, Eye and ENT Hospital, College of Medicine, Fudan University, Shanghai 200032, China. ${ }^{2}$ Shanghai Key Laboratory of Visual Impairment and Restoration, Science and Technology Commission of Shanghai Municipality, Shanghai, China. ${ }^{3}$ BGI-Shenzhen, Shenzhen, Guangdong, China. ${ }^{4}$ Key Laboratory of Myopia (Fudan University), Chinese Academy of Medical Sciences, National Health Commission, Yangpu District, China. ${ }^{5}$ Laboratory of Genomics and Molecular Biomedicine, Department of Biology, University of Copenhagen, Copenhagen, Denmark. ${ }^{6}$ Department of Computer Science, City University of Hong Kong, Kowloon, Hong Kong, China. ${ }^{7}$ BGl Education 
Center, University of Chinese Academy of Sciences, Shenzhen, China. ${ }^{8}$ Shenzhen Engineering Laboratory for Birth Defects Screening, BGI-Shenzhen, Shenzhen, China.

Received: 9 December 2019 Accepted: 31 March 2021 Published online: 13 April 2021

\section{References}

1. den Hollander Al, Roepman R, Koenekoop RK, Cremers FP. Leber congenital amaurosis: genes, proteins and disease mechanisms. Prog Retin Eye Res. 2008;27(4):391-419.

2. Astuti GD, Bertelsen M, Preising MN, Ajmal M, Lorenz B, Faradz SM, Qamar R, Collin RW, Rosenberg T, Cremers FP. Comprehensive genotyping reveals RPE65 as the most frequently mutated gene in Leber congenital amaurosis in Denmark. Eur J Hum Genet. 2016;24(7):1071-9.

3. Liu J, Bu J. A gene scan study of RPE65 in Chinese patients with leber congenital amaurosis. Chin Med J. 2017;130(22):2709-12.

4. Wang H, Wang X, Zou X, Xu S, Li H, Soens ZT, Wang K, Li Y, Dong F, Chen $R$, et al. Comprehensive molecular diagnosis of a large Chinese leber congenital amaurosis cohort. Invest Ophthalmol Vis Sci. 2015;56(6):3642-55.

5. Chung DC, Bertelsen M, Lorenz B, Pennesi ME, Leroy BP, Hamel CP, Pierce E, Sallum J, Larsen M, Stieger K, et al. The natural history of inherited retinal dystrophy due to biallelic mutations in the RPE65 gene. Am J Ophthalmol. 2018;199:58-70.

6. Hull S, Mukherjee R, Holder GE, Moore AT, Webster AR. The clinical features of retinal disease due to a dominant mutation in RPE65. Mol Vis. 2016;22:626-35.

7. Gao FJ, Li JK, Chen H, Hu FY, Zhang SH, Qi YH, Xu P, Wang DD, Wang LS, Chang Q, et al. Genetic and clinical findings in a large cohort of Chinese patients with suspected retinitis pigmentosa. Ophthalmology. 2019:126:1549-56.

8. Zhong Z, Rong F, Dai Y, Yibulayin A, Zeng L, Liao J, Wang L, Huang Z, Zhou Z, Chen J. Seven novel variants expand the spectrum of RPE65related Leber congenital amaurosis in the Chinese population. Mol Vis. 2019;25:204-14

9. Yang G, Liu Z, Xie S, Li C, Lv L, Zhang M, Zhao J. Genetic and phenotypic characteristics of four Chinese families with fundus albipunctatus. Sci Rep. 2017;7:46285.

10. Jacobson SG, Cideciyan AV, Aleman TS, Sumaroka A, Schwartz SB, Windsor EA, Roman AJ, Heon E, Stone EM, Thompson DA. RDH12 and RPE65, visual cycle genes causing leber congenital amaurosis, differ in disease expression. Invest Ophthalmol Vis Sci. 2007:48(1):332-8.

11. Roman AJ, Cideciyan AV, Schwartz SB, Olivares MB, Heon E, Jacobson SG. Intervisit variability of visual parameters in Leber congenital amaurosis caused by RPE65 mutations. Invest Ophthalmol Vis Sci. 2013:54(2):1378-83

12. El Matri L, Ambresin A, Schorderet DF, Kawasaki A, Seeliger MW, Wenzel A, Arsenijevic Y, Borruat FX, Munier FL. Phenotype of three consanguineous Tunisian families with early-onset retinal degeneration caused by an R91W homozygous mutation in the RPE65 gene. Graefe's Arch Clin Exp Ophthalmol. 2006;244(9):1104-12

13. Li S, Xiao X, Yi Z, Sun W, Wang P, Zhang Q. RPE65 mutation frequency and phenotypic variation according to exome sequencing in a tertiary centre for genetic eye diseases in China. Acta Ophthalmol. 2019;98:e181-90.

14. Philp AR, Jin M, Li S, Schindler El, lannaccone A, Lam BL, Weleber RG, Fishman GA, Jacobson SG, Mullins RF, et al. Predicting the pathogenicity of RPE65 mutations. Hum Mutat. 2009;30(8):1183-8.

15. Huang XF, Huang F, Wu KC, Wu J, Chen J, Pang CP, Lu F, Qu J, Jin ZB. Genotype-phenotype correlation and mutation spectrum in a large cohort of patients with inherited retinal dystrophy revealed by next-generation sequencing. Genet Med. 2015;17(4):271-8.
16. Thompson DA, Gyurus P, Fleischer LL, Bingham EL, McHenry CL, Apfelstedt-Sylla E, Zrenner E, Lorenz B, Richards JE, Jacobson SG, et al. Genetics and phenotypes of RPE65 mutations in inherited retinal degeneration. Invest Ophthalmol Vis Sci. 2000;41(13):4293-9.

17. Morimura H, Fishman GA, Grover SA, Fulton AB, Berson EL, Dryja TP. Mutations in the RPE65 gene in patients with autosomal recessive retinitis pigmentosa or leber congenital amaurosis. Proc Natl Acad Sci USA. 1998;95(6):3088-93.

18. Takahashi Y, Chen Y, Moiseyev G, Ma JX. Two point mutations of RPE65 from patients with retinal dystrophies decrease the stability of RPE65 protein and abolish its isomerohydrolase activity. J Biol Chem. 2006;281(31):21820-6.

19. Soens ZT, Branch J, Wu S, Yuan Z, Li Y, Li H, Wang K, Xu M, Rajan L, Motta FL. Leveraging splice-affecting variant predictors and a minigene validation system to identify Mendelian disease-causing variants among exon-captured variants of uncertain significance. Hum Mutat. 2017;38(11):1521-33.

20. Li S, Hu J, Jin RJ, Aiyar A, Jacobson SG, Bok D, Jin M. Temperature-sensitive retinoid isomerase activity of RPE65 mutants associated with Leber Congenital Amaurosis. J Biochem. 2015:158(2):115-25.

21. Kondo H, Qin M, Mizota A, Kondo M, Hayashi H, Hayashi K, Oshima K, Tahira T, Hayashi K. A homozygosity-based search for mutations in patients with autosomal recessive retinitis pigmentosa, using microsatellite markers. Invest Ophthalmol Vis Sci. 2004;45(12):4433-9.

22. Jacobson SG, Aleman TS, Cideciyan AV, Sumaroka A, Schwartz SB, Windsor EA, Traboulsi El, Heon E, Pittler SJ, Milam AH, et al. Identifying photoreceptors in blind eyes caused by RPE65 mutations: prerequisite for human gene therapy success. Proc Natl Acad Sci USA. 2005;102(17):6177-82.

23. Hamel CP, Griffoin JM, Bazalgette C, Lasquellec L, Duval PA, Bareil C, Beaufrere L, Bonnet S, Eliaou C, Marlhens F, et al. Molecular genetics of pigmentary retinopathies: identification of mutations in $\mathrm{CHM}, \mathrm{RDS}, \mathrm{RHO}$, RPE65, USH2A and XLRS1 genes. J Fr Ophtalmol. 2000;23(10):985-95.

24. Mo G, Ding Q, Chen Z, Li Y, Yan M, Bu L, Song Y, Yin G. A novel mutation in the RPE65 gene causing Leber congenital amaurosis and its transcriptional expression in vitro. PLOS ONE. 2014;9(11):e112400.

25. Li L, Xiao X, Li S, Jia X, Wang P, Guo X, Jiao X, Zhang Q, Hejtmancik JF. Detection of variants in 15 genes in 87 unrelated Chinese patients with Leber congenital amaurosis. PLoS ONE. 2011;6(5):e19458.

26. Simovich MJ, Miller B, Ezzeldin H, Kirkland BT, McLeod G, Fulmer C, Nathans J, Jacobson SG, Pittler SJ. Four novel mutations in the RPE65 gene in patients with Leber congenital amaurosis. Hum Mutat. 2001;18(2):164.

27. Xu F, Dong Q, Liu L, Li H, Liang X, Jiang R, Sui R, Dong F. Novel RPE65 mutations associated with Leber congenital amaurosis in Chinese patients. Mol Vis. 2012;18:744-50.

28. Fu Q, Wang F, Wang H, Xu F, Zaneveld JE, Ren HN, Keser V, Lopez I, Tuan HF, Salvo JS, et al. Next-generation sequencing-based molecular diagnosis of a Chinese patient cohort with autosomal recessive retinitis pigmentosa. Invest Ophthalmol Vis Sci. 2013;54(6):4158-66.

29. Sitorus RS, Lorenz B, Preising MN. Analysis of three genes in Leber congenital amaurosis in Indonesian patients. Vis Res. 2003;43(28):3087-93.

30. Zernant J, Kulm M, Dharmaraj S, den Hollander Al, Perrault I, Preising MN, Lorenz B, Kaplan J, Cremers FP, Maumenee I, et al. Genotyping microarray (disease chip) for Leber congenital amaurosis: detection of modifier alleles. Invest Ophthalmol Vis Sci. 2005:46(9):3052-9.

31. Stone EM. Leber congenital amaurosis-a model for efficient genetic testing of heterogeneous disorders: LXIV Edward Jackson Memorial Lecture. Am J Ophthalmol. 2007;144(6):791-811.

\section{Publisher's Note}

Springer Nature remains neutral with regard to jurisdictional claims in published maps and institutional affiliations. 